\title{
1 Schizophrenia risk from locus-specific human endogenous retroviruses
}

3 Rodrigo R.R. Duarte ${ }^{a-c}$, Matthew L. Bendall ${ }^{c, d}$, Miguel de Mulder ${ }^{c}$, Christopher E. Ormsbye, Greta A.

4 Beckerle $^{c}$, Sashika Selvackadunco ${ }^{\dagger}$, Claire Troakes $^{\dagger}$, Gustavo Reyes-Terán ${ }^{e}$, Keith A. Crandall ${ }^{d}$

5 Deepak P. Srivastava ${ }^{\dagger \mathrm{b}, \mathrm{g}}$, Douglas F. Nixon ${ }^{\dagger \mathrm{c}}$ and Timothy R. Powell ${ }^{\star \dagger a, c}$

6 † Shared senior authors.

8 a Social, Genetic \& Developmental Psychiatry Centre, Institute of Psychiatry, Psychology \&

9 Neuroscience, King's College London, London, UK.

$10{ }^{b}$ Department of Basic \& Clinical Neuroscience, Institute of Psychiatry, Psychology \& Neuroscience,

11 King's College London, London, UK.

$12{ }^{c}$ Division of Infectious Diseases, Weill Cornell Medicine, Cornell University, New York, NY, USA.

$13{ }^{\mathrm{d}}$ Computational Biology Institute, Milken Institute School of Public Health, The George Washington 14 University, Washington, DC, USA.

15 e Center for Research in Infectious Diseases, National Institute of Respiratory Diseases, Mexico City, 16 Mexico.

$17{ }^{f}$ MRC London Neurodegenerative Diseases Brain Bank, Institute of Psychiatry, Psychology \& 18 Neuroscience, King's College London, London, United Kingdom.

19 g MRC Centre for Neurodevelopmental Disorders, King's College London, London, United Kingdom.

21 * Correspondence to: Dr. Timothy R. Powell, Social, Genetic \& Developmental Psychiatry Centre, 22 Institute of Psychiatry, Psychology \& Neuroscience, King's College London, 16 De Crespigny Park, 23 London SE5 8AF, United Kingdom. E-mail: timothy.1.powell@kcl.ac.uk. Tel: +44 (0)20 78485361. 


\section{Abstract}

25 Schizophrenia genome-wide association studies highlight the substantial contribution of risk attributed to the non-coding genome where human endogenous retroviruses (HERVs) are encoded.

27 These ancient viral elements have previously been overlooked in genetic and transcriptomic studies

28 due to their poor annotation and repetitive nature. Using a new, comprehensive HERV annotation,

29 we found that the fraction of the genome where HERVs are located (the 'retrogenome') is enriched

30 for schizophrenia risk variants, and that there are 148 disparate HERVs involved in susceptibility.

31 Analysis of RNA-sequencing data from the dorsolateral prefrontal cortex of 259 schizophrenia cases

32 and 279 controls from the CommonMind Consortium showed that HERVs are actively expressed in

33 the brain ( $n=3,979)$, regulated in cis by common genetic variants ( $n=1,759)$, and differentially

34 expressed in patients $(n=81)$. Convergent analyses implicate LTR25_6q21 and ERVLE_8q24.3h

35 as HERVs of etiological relevance to schizophrenia, which are co-regulated with genes involved in

36 neuronal and mitochondrial function, respectively. Our findings provide a strong rationale for 37 exploring the retrogenome and the expression of these locus-specific HERVs as novel risk factors 38 for schizophrenia and potential diagnostic biomarkers and treatment targets. 
bioRxiv preprint doi: https://doi.org/10.1101/798017; this version posted October 8, 2019. The copyright holder for this preprint (which was not certified by peer review) is the author/funder, who has granted bioRxiv a license to display the preprint in perpetuity. It is made available under aCC-BY-NC 4.0 International license.

\section{Introduction}

Human endogenous retroviruses (HERVs) are remnants of genetic material acquired through our evolutionary past which originated from the infection of germline cells with ancient retroviruses. These viruses multiplied through a copy-and-paste mechanism and were eventually endogenized (i.e., vertically transmitted), and now constitute approximately $8 \%$ of the genome ${ }^{1,2}$. These repetitive sequences were generally assumed to be transcriptionally inactive in the modern genome, having a purely regulatory function due to the retainment of the viral promoters (long-terminal repeats, LTRs). However, certain HERVs were co-opted to serve novel specialized roles, including in the regulation of embryonic development ${ }^{3,4}$ and neural progenitor cells $s^{5,6}$. They have also been implicated in neuropsychiatric conditions such as amyotrophic lateral sclerosis ${ }^{7-9}$, major depressive disorder, bipolar disorder, and schizophrenia ${ }^{10-12}$. Despite their abundance in the genome and relevance to disease and fundamental aspects of human biology, the location and function of most HERVs remain elusive to-date.

Recent large genome-wide association studies (GWAS) comparing schizophrenia cases and nonaffected individuals enabled the identification of polymorphisms mediating risk for this disorder ${ }^{13-15}$. These studies highlight the substantial contribution of risk attributed to the non-coding genome, where HERVs are encoded, which are often overlooked in both genomic and transcriptomic studies $^{16}$. Until recently, there was no comprehensively annotated map of HERVs in the genome, and there were no computationally efficient tools to analyze the expression of these repetitive sequences with single-locus resolution. Consequently, previous studies were unable to test whether locus-specific HERVs were genetically associated with traits of interest, or to assess their expression in conditions of interest, or to distinguish expressed from dormant HERVs from within the same family, which contributed to the generation of inconclusive findings. For example, Karlsson and colleagues ${ }^{17}$ reported decreased ERV9 expression in the brain of schizophrenia patients, whereas Diem and collaborators ${ }^{18}$ found the opposite. While the heterogeneity of schizophrenia is also likely to be a contributing factor for these contradictory findings, there are approximately 120 copies of ERV9 in the genome ${ }^{19,20}$ that likely also confounded these reports. 
69 Recently, there have been significant advances in the annotation of HERVs in the genome ${ }^{20-24}$, and

70 in the development of tools that are able to map repetitive sequences in RNA-sequencing data to

71 their most likely source of origin in the genome $20,25,26$. These improvements allow for the identification

72 and quantification of specific HERV copies associated with traits of interest. These advances,

73 combined with modern population genetic methods, enabled us to identify the contribution of the

74 'retrogenome' (the full genomic complement of HERVs) and expression of locus-specific HERVs to

75 schizophrenia etiology and neurobiology. 
bioRxiv preprint doi: https://doi org/10.1101/798017; this version posted October 8 2019. The copyright holder for this preprint (which was not certified by peer review) is the author/funder, who has granted bioRxiv a license to display the preprint in perpetuity. It is made available under aCC-BY-NC 4.0 International license.

\section{Results}

\section{The contribution of polymorphisms in the retrogenome to schizophrenia risk}

A recently developed annotation of putatively functional HERV elements describes 14,968 elements dispersed throughout the genome, which were defined based on the presence of LTRs and remnants of genetic elements which code for viral proteins like env, gag or popo. We assessed the contribution of common genetic variants within HERVs to schizophrenia susceptibility using GARFIELD ${ }^{27}$. This pipeline quantifies the co-localization of GWAS results with variants in annotation categories (i.e. variants in the retrogenome), assessing significance using linear models that control for minor allele frequency and linkage disequilibrium. For comparison, enrichment was also calculated for heritable neuropsychiatric traits and non-neuropsychiatric phenotypes: height, body mass index, coronary artery disease, Crohn's Disease, type 2 diabetes, neuroticism, eczema, major depressive disorder, bipolar disorder, Alzheimer's disease, attention deficit hyperactivity disorder, amyotrophic lateral sclerosis, and autism spectrum disorder (Figure 1; full results on Supplemental Table 1). Polymorphisms within HERVs were significantly enriched for genome-wide significant variants $(\mathrm{P}<$ $\left.5.00 \times 10^{-8}\right)$ associated with schizophrenia $\left(P_{\text {enrichment }}=1.88 \times 10^{-5}, P_{\text {corrected }}\right.$ [for 14 traits and 2 GWAS thresholds tested $\left.]=5.27 \times 10^{-4}, \beta=0.90,95 \% \mathrm{Cl}[0.49,1.31]\right)$. Analysis of variants associated with these traits under a more relaxed $P$ cut-off $\left(P<5.00 \times 10^{-5}\right)$ also showed an enrichment for schizophrenia only $\left(P_{\text {enrichment }}=4.56 \times 10^{-7}, P_{\text {corrected }}=1.28 \times 10^{-5}, \beta=0.57,95 \% \mathrm{Cl}[0.35,0.80]\right)$. These findings suggest a role for the retrogenome in the etiology of this neurodevelopmental disorder.

$<<<$ Figure $1>>>$

\section{The contribution of locus-specific HERVs and HERV families to schizophrenia susceptibility}

We co-localized polymorphisms within individual HERVs and those associated with schizophrenia by GWAS using MAGMA ${ }^{28}$, which calculates gene-level statistics and weighted $p$-values based on summary statistics whilst adjusting for gene size, single nucleotide polymorphism (SNP) density and linkage disequilibrium. This gene-level enrichment analysis revealed that $148 \mathrm{HERVs}$ from multiple families were significantly enriched for risk variants implicated in schizophrenia, after correcting for 
105 the number of HERVs tested $\left(P_{\text {corrected }}<0.05 / 12,389\right.$ HERVs in chromosomes 1-22, excluding those at the major histocompatibility locus; Supplemental Table 2). The quantile-quantile plot highlights the contribution of many locus-specific HERVs associated with risk, compared to an expected normal distribution (Figure 2A), and the Manhattan plot shows their diverse genomic location (Figure 2B).

To explore the contribution of HERV families towards schizophrenia risk, we investigated whether

111 any of the 60 families defined in the HERV annotation were overrepresented in the list of schizophrenia-associated HERVs using a gene-set enrichment analysis in MAGMA. Each HERV was assigned to a family based on the RepBase model that most closely matched the internal region sequences $^{20,29}$. We observed a nominal association between schizophrenia and the HERVL40 family $(P=0.02, \beta=0.14 \pm 0.02, S E=0.07)$, but this did not survive multiple testing correction $\left(P_{\text {corrected }}\right.$

116 [for 60 families] > 0.05; Supplemental Figure 1). These findings suggest that risk for schizophrenia 117 attributed to the retrogenome may occur via locus-specific sequences, as opposed to entire HERV 118 families.

\section{HERV expression in the dorsolateral prefrontal cortex}

123 To advance our understanding of HERV expression in the adult brain, we assessed global HERV 124 expression (the retrotranscriptome) in the dorsolateral prefrontal cortex (DLPFC) of 593 post-mortem individuals ( $N=593$ ), including 279 unaffected controls, 259 schizophrenia patients, 47 bipolar disorder patients and 8 cases broadly diagnosed with an affective disorder. This was achieved by applying the Telescope pipeline ${ }^{20}$ to the RNA-seq data from the CommonMind Consortium (CMC) dataset. Analysis of these samples revealed that 3,979 HERVs were consistently expressed in the

129 DLPFC according to DESeq2 independent filtering criteria (mean normalized counts $=40.79$ [37.8,

130 43.78]; Figure 3A). We performed RT-qPCR to confirm the expression of five arbitrarily selected 131 HERVs in an independent post-mortem cohort of control individuals from the London 132 Neurodegenerative Diseases Brain Bank ( $N=10$; Supplemental Material; Supplemental Figure 2). 


\section{Cis-acting HERV eQTLs in the DLPFC}

139 To understand how HERVs are regulated in the brain, we performed an expression quantitative trait 140 loci (eQTL) analysis using all samples $(\mathrm{N}=593)$. Such analysis aims to identify SNPs that explain 141 variation in the expression of HERVs residing in close proximity, to inform about the basic processes responsible for HERV regulation, and to complement our genetic enrichment analyses. The genomic coordinates from the expressed HERVs were remapped to hg19 positions to match genotype information, and only HERVs from chromosomes 1-22 were analyzed (total = 5,349 HERVs, which includes lowly expressed HERVs, according to DESeq2's internal filtering criteria). This analysis revealed that 1,759 HERVs were regulated in cis by 1,622 SNPs located within a $1 \mathrm{Mb}$ window upstream or downstream the start site of the HERV annotation, under the false discovery rate of $5 \%$ $(q<0.05)$. The majority of eQTLs were located within a $10 \mathrm{~kb}$ window upstream or downstream of the annotation start site from the HERV they regulate (Figure 3B). Of the 148 HERVs that were enriched for schizophrenia variants, we observed that 19 were significantly regulated by eQTLs in the DLPFC (Supplemental Tables 2 and 3, highlighted in green; Figure 3C).

\section{Case-control differences in HERV expression}

154 We observed $81 \mathrm{HERVs}$ as differentially expressed between cases $(\mathrm{N}=259)$ and unaffected individuals $(\mathrm{N}=279)$ under the false discovery rate of $5 \%$, independent of a genetic association with schizophrenia $(q<0.05$ [corrected for the 3,979 expressed HERVs]; Supplemental Figure 4,

157 Supplemental Table 4). Our analysis showed that 36 HERVs were downregulated and 45 158 upregulated in cases, and that expression differences were subtle, with log2 fold-changes of $0.18 \pm$ 1590.10 on average. 
163 To make a more informative assessment of HERV expression differences associated with disease

164 status, we first explored whether HERVs identified as genetic risk factors for this disorder were expressed in the DLPFC. Of the $148 \mathrm{HERV}$ identified via gene-level enrichment analysis, only 65 were consistently expressed across samples according to DESeq2 internal filtering criteria, with mean normalized counts of $57.5, \mathrm{Cl} 95 \%$ [12.59, 102.3]. Of these, we observed that two were significantly upregulated (ERV316A3_12q24.11, log2 fold-change $=0.16$, standard error $=0.06$; LTR25_6q21, log2 fold-change $=0.09$, standard error $=0.04)$, and one downregulated $($ ERVLE_8q24.3h; log2 fold-change $=-0.09$, standard error $=0.04)$ in schizophrenia cases relative to unaffected controls, under the false discovery rate of $5 \%(q<0.05$ [corrected for $65 \mathrm{HERVs}$; Figures 3C and D; Supplemental Table 5).

Of the three HERVs enriched for schizophrenia variants and differentially expressed in cases, we observed that two were modulated by eQTLs (Figures $\mathbf{3 C}$ and D, highlighted in blue), which we initially hypothesized could explain the case-control differences observed. These included ERVLE_8q24.3h and LTR25_6q21, which were regulated by the top eQTLs, rs4875048 and rs174399, respectively. Importantly, rs4875048 is associated with schizophrenia and is in linkage disequilibrium with the top association signal at this locus, rs10552126; rs174399 is in linkage 180 disequilibrium with a risk variant at the locus, rs 11153302 (Table 1) ${ }^{14}$. Strikingly, no gene within a 1 $181 \mathrm{Mb}$ window upstream or downstream of either of the two top eQTLs was associated with schizophrenia according to a recent analysis by

PsychENCODE (http://resource.psychencode.org/) ${ }^{30}$.

We observed complex regulatory mechanisms governing expression of these two HERVs. ERVLE_8q24.3h was significantly downregulated in cases, but, contrary to what was expected, the risk (A-) allele of rs4875048 was associated with increased expression of this HERV $\left(\beta=-0.35, P_{\beta}\right.$ dist. $=0.002, q=0.007$, Figure 4A). Similarly, LTR25_6q21 was upregulated in patients, but the risk

(G-) allele of rs174399 was associated with reduced expression of this HERV $(\beta=0.29$, $190 P_{\beta \text { dist. }}=0.004, q=0.02$; Figure 4B). We investigated the effect of these eQTLs in cases and control 191 individuals separately, which revealed that they influenced HERV expression exclusively in 
192 unaffected individuals, suggesting there is a compensatory mechanism in patients counteracting the

193 effects of the eQTLs (Figures 4A and B; Supplemental Table 6).

195 To understand about the regulation of these HERVs during neurodevelopment, we tested their 196 expression in an in vitro model of cortical development, which consisted of neural stem cells from 197 the CTX0E16 cell line and cells differentiated for 28 days ${ }^{31-33}$ (Supplemental Material, 198 Supplemental Figure 5). We observed that both HERVs were differentially regulated during 199 differentiation, suggesting that risk to schizophrenia pertaining these HERVs starts during 200 neurodevelopment. Interestingly, we also observed that both HERVs are located in the antisense 201 strand of SLC16A10 and IQANK1 introns, respectively. Moreover, LTR25_6q21 is exclusively 202 present in humans, whereas ERVLE_8q24.3h is shared with primates (Supplemental Figure 6), 203 according to the UCSC Genome Browser ${ }^{34}$.

ERVLE_8q24.3h and LTR25_6q21 are co-regulated with genes implicated in mitochondrial and synaptic function in the adult brain, respectively

To explore the potential function of ERVLE_8q24.3h and LTR25_6q21, we determined the genes

211 that are co-expressed with these HERVs by applying Weighted Correlation Network Analysis 212 (WGCNA $)^{35}$ to the RNA-seq data from schizophrenia patients and controls combined. This systems 213 biology approach is based on the hypothesis that genes within the same co-regulated network share 214 the same function ${ }^{36}$. We observed 19 modules of co-expression in these data (Supplemental 215 Tables 7 and 8, Supplemental Figure 7). LTR25_6q21 was assigned to the green module (module 216 membership statistic $(\mathrm{MM})=0.41, \mathrm{P}=3.55 \times 10^{-23}$; gene significance (GS) statistic in relation to 217 case-control status: 0.12 , GS $P=0.005)$, whereas ERVLE_8q24.3h was assigned to the turquoise 218 module $\left(\mathrm{MM}=0.46, \mathrm{P}=4.81 \times 10^{-29} ; \mathrm{GS}=-0.15\right.$, GS $\left.\mathrm{P}=5.14 \times 10^{-4}\right)$. Gene Ontology (GO) analysis 219 of the green module indicates that this gene set is associated with neuronal function, with significant terms including "synapse organization", "presynapse" and "vesicle-mediated transport in synapse" 
221 ( $q<0.05$, Figure 5A, upper panel, Supplemental Table 9). The turquoise module, in turn, was significantly associated with mitochondrial function, with terms including the "respiratory chain" and "mitochondrial matrix", and "NADH dehydrogenase complex assembly" ( $q<0.05$, Figure 5A, lower panel, Supplemental Table 9).

A correlation between the first principal component capturing the variability within each module (module eigengene) and case-control status ('Profile') was performed in WGCNA, which revealed that the green module is positively associated with case-control status $(r=0.13, P=0.003)$, whereas the turquoise module was negatively associated $\left(r=-0.15, P=4 \times 10^{-4}\right.$; Figure 5B). The coexpression modules were detected assuming a signed network, which means that a positive module-

231 trait correlation entails higher expression of genes in the module in association with disease status, 232 and vice-versa, corroborating the case-control differences observed for each HERV in the previous 233 analysis (Figures 4A and B, left panels).

To understand the function of other HERVs associated with schizophrenia from the gene-level enrichment analysis performed using MAGMA (Supplemental Table 2), we identified the coexpression modules associated with each enriched HERV that was expressed in the brain

238 (Supplemental Table 10). We observed that several of these HERVs were assigned to modules 239 implicated in synaptic organization, including MER41_1p33 and MER41_5p12 alongside LTR25_6q21 in the green module, as well as synaptic function, including HERVL_14q24.2d, HERVL40_8q21.3b, HERVW_6q21c, HML3_5p12a and MER41_2q31.2 in the red module. Interestingly, we observed that these two modules were clustered together in an unsupervised 243 hierarchical clustering analysis performed in WGCNA, corroborating a related or shared function 244 (Supplemental Figure 8). 
250 We investigated the major cell types associated with each modules eigengene (the first principal 251 component of the module) to infer the cell types associated with ERVLE_8q24 and LTR25_6q21. 252 We estimated the proportion of neural cell types in the RNA-sequencing data by applying the R package BRETIGEA ${ }^{37}$ to the normalized gene counts of the RNA-sequencing data. BRETIGEA

254 estimates the cell type proportions that constitute the sequenced material based on a database of 255 single-cell RNA-sequencing data, ultimately generating coefficients that represent the proportion of 256 astrocytes, microglia, endothelial cells, oligodendrocytes, oligodendrocyte progenitor cells and 257 neurons in the sequenced samples ${ }^{37}$. To infer the cell types associated with each module, we 258 performed correlations between each module and the cell-type proportion coefficients (Figure 5B). 259 The module assigned to LTR25_6q21 (green) was significantly correlated with the expression of 260 neuronal markers $\left(r=0.24, P=3 \times 10^{-8}\right)$, and negatively associated with the expression of all other 261 cell types ( $q<0.05$, corrected for the six cell types tested and the two modules of interest), consistent 262 with a role for this module in the regulation of neuronal function, as indicated by the GO analysis. 263 The module assigned to ERVLE_8q24.3h (turquoise) was negatively correlated with the expression 264 of oligodendrocyte progenitor cell markers $\left(r=-0.21, P=6 \times 10^{-7}, q<0.05\right)$, but showed no 265 correlation with the other cell types, which may suggest a specific role for this HERV in non-dividing 266 cells. Ultimately, these data indicate that LTR25_6q21 and ERVLE_8q24.3h are part of co-regulated 267 networks implicated in neuronal and mitochondrial function, respectively, suggesting their 268 involvement in these cell functions. 
bioRxiv preprint doi: https://doi.org/10.1101/798017; this version posted October 8, 2019. The copyright holder for this preprint (which was not certified by peer review) is the author/funder, who has granted bioRxiv a license to display the preprint in perpetuity. It is made available under aCC-BY-NC 4.0 International license.

\section{Discussion}

HERVs are ancient viral genetic elements scattered throughout the genome, with previously hypothesized influences on neurodevelopment and risk for schizophrenia. We took a comprehensive approach to reconsider the role of HERVs at the omics level, leveraging on the recent advances in the genomic annotation of HERVs, single-locus resolution quantification, systems biology methods, and population genetic tools.

We investigated the combined contribution of HERVs (the retrogenome) to risk for schizophrenia and other complex polygenic traits, by assessing the overlap between SNPs implicated in these traits and those in genomic locations encompassing HERVs. We were surprised to find that schizophrenia was the only tested trait significantly enriched for common variants within the retrogenome, especially since there is evidence linking HERVs to conditions like amyotrophic lateral sclerosis ${ }^{7-9}$, Crohn's disease ${ }^{38}$, major depressive disorder and bipolar disorder ${ }^{10,11}$. The fact that the retrogenome is significantly enriched for polymorphisms implicated in schizophrenia suggests that HERVs comprise an important set of risk factors for this disorder, within the 'non-coding' genome.

We investigated which HERV families and locus-specific HERVs could be particularly important in moderating risk for schizophrenia at the genetic level by co-localizing risk variants with individual HERV loci from across 60 families. We identified 148 specific HERVs enriched for schizophreniaassociated SNPs. A subsequent gene-set analysis did not find a particular HERV family enriched for schizophrenia variants, further suggesting that disparate HERVs scattered throughout the genome moderate susceptibility rather than whole families. These findings have several implications for the interpretation of previous studies in the literature, and may explain how our results differ from previous data implicating other HERVs or HERV families as risk factors, which may not have been identified here. Most HERV expression research to-date has been performed using microarrays, antibodies or RT-qPCR probes, which do not provide sufficient specificity to assess single HERVs ${ }^{7-}$ 11. Therefore, previous studies may have captured the expression of multiple HERVs concomitantly due to their repetitive sequences. Based on our findings, it is unlikely that individual HERVs (even from within the same family) contribute equally to risk. The HERV annotation developed by Bendall 
bioRxiv preprint doi: https://doi.org/10.1101/798017; this version posted October 8, 2019. The copyright holder for this preprint (which was not certified by peer review) is the author/funder, who has granted bioRxiv a license to display the preprint in perpetuity. It is made available under aCC-BY-NC 4.0 International license.

301 and colleagues $^{20}$, as well as the Telescope pipeline, and the application of modern population genetic methods, enabled us to revisit the role HERVs play in relation to schizophrenia risk on a genome-wide scale, and allow for more robust inferences regarding their etiological relevance.

Next, to better understand HERV expression regulation in the brain, we analyzed RNA-sequencing data from the CommonMind Consortium, which confirmed widespread HERV expression in the adult brain and cis-regulatory mechanisms. We found 3,979 HERVs actively expressed in a key brain area linked to schizophrenia pathophysiology, the DLPFC ${ }^{39}$. We further identified 1,759 HERVs that were regulated by 1,622 short-range (cis-) eQTLs in the DLPFC. The identification of eQTLs that impact HERV expression informs us about the basic processes responsible for HERV regulation, and can

311 be useful for the interpretation of GWAS findings in the context of the retrogenome $e^{40,41}$. In addition,

312 these results suggest that SNPs within HERVs are not simply affecting the expression of neighboring 313 protein-coding genes via their LTRs, rather it demonstrates that common genetic variation impacts 314 locally on HERV expression.

We used a complementary set of analyses to identify the most robust HERVs implicated in 317 schizophrenia. Two HERVs, ERVLE_8q24.3h and LTR25_6q21, identified from the gene-level 318 enrichment analysis, were found to be regulated by schizophrenia-associated eQTLs, and were 319 found to be differentially expressed in the DLPFC of patients and in an in vitro model of cortical neurodevelopment. These findings suggest a potential risk mechanism for schizophrenia that starts during neurodevelopment and persists through to adulthood, as observed for schizophrenia risk genes such as NT5C2, AS3MT, and BORCS $7^{33,42}$. Importantly, we observed a complex regulation of both HERVs, whereby the lead eQTLs only exerted their regulatory effects in unaffected individuals. This suggests that compensatory mechanisms (e.g. epigenetic alterations) may be acting to correct for the effects of the risk variants on the expression of these HERVs in the DLPFC of schizophrenia patients.

We also describe here, for the first time, the co-regulation of several HERVs with known genes in the adult brain, and the GO terms associated with each co-expression module. Our findings suggest 
bioRxiv preprint doi: https://doi.org/10.1101/798017; this version posted October 8, 2019. The copyright holder for this preprint (which was not certified by peer review) is the author/funder, who has granted bioRxiv a license to display the preprint in perpetuity. It is made available under aCC-BY-NC 4.0 International license.

330 that LTR25_6q21 is implicated in neuronal function, whereas ERVLE_8q24.3h is involved in

331 mitochondrial function. WGCNA has been successfully used to predict the biological function of 332 unknown genes or non-coding RNAs in different organisms ${ }^{43,44}$, and to identify clinically relevant cell 333 types when in combination with cell-type deconvolution analysis ${ }^{45}$, and thus represents a powerful 334 approach to functionally characterize the HERVs expressed in the brain. For a long time HERVs 335 were assumed to be mere regulatory DNA sequences, but the discovery of their expression and co336 regulation with several other genes implicated in multiple biological processes in the brain, ranging 337 from neuronal, glial and mitochondrial regulation to splicing and cell motility (Supplemental Table 338 9), is a landmark for HERV research, and adds an extra layer of complexity to our understanding of 339 human neurobiology.

341 There are limitations to this study which should be acknowledged. Schizophrenia is a highly 342 polygenic, heterogeneous disorder, and as such large sample sizes are required for appropriate 343 comparisons. The CommonMind Consortium provides the largest and best characterized cohort of 344 schizophrenia cases and unaffected individuals with RNA-sequencing data to-date, but it might be 345 underpowered for case-control comparisons considering the heterogeneity of schizophrenia. 346 Nevertheless, we complemented case-control comparisons with genomic and eQTL analyses to 347 provide additional insights. Another limitation to our study is that it investigated RNA-sequencing 348 data from bulk DLPFC tissue only, which is composed of a heterogeneous mixture of several types 349 of neurons and glial cells, and it is possible that HERVs expressed in particular cell types, or in other 350 brain regions, are more relevant to risk $^{46}$. To address this, we performed cell type deconvolution 351 using BRETIGEA to determine cell-type specific effects, but ultimately the analysis of data from other 352 brain areas, developmental time points and single-cell datasets has the potential to reveal important 353 insights about the etiology of schizophrenia in relation to HERV expression. In addition, our post354 mortem and in vitro work suggest HERV expression is important in the DLPFC and during its 355 development, but we still do not know the function of these HERVs. To infer function we performed 356 WGCNA which provides insight into which processes risk HERVs moderate, but future functional 357 studies are required to definitively characterize how HERVs, particularly ERVLE_8q24.3h and LTR25_6q21, influence the transcriptome, neural stem cell proliferation or neuronal differentiation in 
359 the context of schizophrenia risk, as is currently being investigated in relation to protein-coding risk 360 genes $^{32,33,47,48}$.

362 The development of a retrogenome annotation, and advances in modern population genetic methods

363 and transcriptomic tools, now allows us to investigate HERVs at the omics level, in the context of 364 risk for many biological traits. Our work studying the role of HERVs in the brain, and their relationship 365 to schizophrenia ignites a new, provocative line of thought implicating HERVs as biological risk 366 factors for schizophrenia and confirms that these previously assumed 'dormant' sequences in the brain may not be dormant after all. 
bioRxiv preprint doi: https://doi org/10.1101/798017; this version posted October 8, 2019. The copyright holder for this preprint (which was not certified by peer review) is the author/funder, who has granted bioRxiv a license to display the preprint in perpetuity. It is made available under aCC-BY-NC 4.0 International license.

\section{Online Methods}

We used a combination of gene expression, genetic and in vitro analyses to identify the most robust HERVs implicated in schizophrenia risk (Figure 6). Further details are provided in the Supplemental

\section{Material.}

\section{$<<$ Figure $6>>$}

\section{Genetic enrichment analyses}

We estimated the contribution of genetic polymorphisms within the retrogenome towards risk of developing multiple traits using GARFIELD ${ }^{27}$. We downloaded summary statistics from well-powered genome-wide association studies, including of schizophrenia $(N=105,318 \text { individuals })^{14}$, height ( $N$ $=693,529)^{49}$, body mass index $(\mathrm{N}=681,275)^{49}$, coronary artery disease $(\mathrm{N}=547,261)^{50}$, Crohn's Disease $(N=59,957)^{51}$, type 2 diabetes $(N=659,316)^{52}$, neuroticism $(N=2,370,390)^{53}$, eczema $(N$ $=103,066)^{54}$, major depressive disorder $(\mathrm{N}=480,359)^{55}$, bipolar disorder $(\mathrm{N}=51,710)^{56}$, Alzheimer's disease $(N=74,046)^{57}$, attention deficit hyperactivity disorder $(N=53,293)^{58}$, amyotrophic lateral sclerosis $(\mathrm{N}=36,052)^{59}$, and autism spectrum disorder $(\mathrm{N}=46,350)^{60}$, which analyzed European cohorts only. GARFIELD performs greedy pruning of SNPs in GWAS summary statistics (those in linkage disequilibrium, with $R^{2}>0.1$ ), and quantifies enrichments using odds ratios, assessing their significance by employing generalized linear model testing, controlling for minor allele frequency, and number of linkage disequilibrium proxies $\left(R^{2}>0.8\right)$. Linkage disequilibrium and allele frequency information were calculated based on the UK10K study. Enrichments were calculated based on summary statistics from each trait using two $P_{\text {association }}$ thresholds: $P<5 \times 10^{-8}$, to test the enrichment of HERVs within genome-wide significant variants; and a more relaxed threshold, $\mathrm{P}<5 \times 10^{-5}$, to allow signal capture in less powered GWAS. The enrichment significance was corrected for the number of tests performed [14 traits and two P-value thresholds tested]. For consistency with the GWAS data, the HERV annotation used in the expression analysis (hg38) was remapped to hg19 coordinates using liftOver ${ }^{34}$.

To identify locus-specific HERVs and potential HERV families associated with schizophrenia, we used MAGMA $1.07 b^{28}$. Briefly, MAGMA calculates gene-level enrichment by generating a gene-wide 
bioRxiv preprint doi: https://doi.org/10.1101/798017; this version posted October 8, 2019. The copyright holder for this preprint (which was not certified by peer review) is the author/funder, who has granted bioRxiv a license to display the preprint in perpetuity. It is made available under aCC-BY-NC 4.0 International license.

statistic from summary statistics, adjusting for gene size, variant density, and linkage disequilibrium using the 1000 Genomes Phase 3 European reference panel. SNPs from the summary statistics were assigned to HERVs using an annotation window of $10 \mathrm{~kb}$ upstream and downstream of each HERV (as suggested by the authors) ${ }^{28}$. A Bonferroni correction was applied to identify significantly enriched HERVs $\left(P_{\text {cut-off }}<4.03 \times 10^{-6}[0.05 / 12,393\right.$ HERVs in chromosomes 1-22, excluding the major histocompatibility locus]). Q-Q and Manhattan plots were generated using qqman $0.1 .4^{61}$. Gene-set enrichment analysis was additionally performed using MAGMA, to test whether HERVs associated with schizophrenia in the previous step were enriched for any of the 60 HERV families (excluding HERVs located in sex chromosomes or at the MHC locus - chromosome 6: 26 - $34 \mathrm{Mb}$ ).

\section{The CommonMind Consortium dataset}

408 To identify HERV expression differences in schizophrenia patients, or HERV expression quantitative 409 trait loci (eQTL) in the dorsolateral prefrontal cortex, we analyzed RNA-sequencing data from the 410 CommonMind Consortium (release 1.0, N = 593 individuals, https://doi.org/10.7303/syn2759792) ${ }^{39}$. 411 This dataset consisted of dorsolateral prefrontal cortex (DLPFC) samples from 279 unaffected 412 individuals, 259 schizophrenia cases, 47 bipolar disorder patients and 8 cases broadly diagnosed 413 with an affective disorder. Access to this dataset, which includes expression, genotype and clinical 414 data, was granted under a Material Transfer Agreement with the NIMH Repository and Genomics 415 Resources. Briefly, autopsy samples from the Mount Sinai NIH Brain Bank and Tissue Repository, 416 the University of Pennsylvania Brain Bank of Psychiatric illnesses and Alzheimer's Disease Core 417 Center, and The University of Pittsburgh Brain Tissue Donation Program, were sent to the Icahn 418 School of Medicine at Mount Sinai for nucleic acid isolation and sequencing. Individuals were 419 diagnosed according to the Diagnostic and Statistical Manual of Mental Disorders, 4th Edition, as 420 determined in consensus conferences after review of medical records and interviews of family 421 members and care providers. Total RNA was extracted from autopsy tissue using the RNeasy kit 422 (QIAGEN, Hilden, Germany). Ribosomal RNA was depleted using the Ribo-Zero Magnetic Gold kit 423 (Illumina, San Diego, California, United States), libraries were constructed using the TruSeq RNA 424 Sample Preparation Kit v2 (Illumina), and samples were sequenced on an Illumina HiSeq 2500. For 
bioRxiv preprint doi: https://doi.org/10.1101/798017; this version posted October 8, 2019. The copyright holder for this preprint (which was not certified by peer review) is the author/funder, who has granted bioRxiv a license to display the preprint in perpetuity. It is made available under aCC-BY-NC 4.0 International license.

according to the manufacturer's protocol, and samples were genotyped using Illumina Infinium HumanOmniExpressExome $81.1 \mathrm{~b}$ chips. Further details on quality control and sample processing are described in Fromer and colleagues ${ }^{39}$.

\section{RNA-sequencing data processing and HERV expression quantification}

Bam files containing mapped and unmapped RNA-sequencing reads aligned to the human reference genome (hg19) using TopHat 2.0.9 and Bowtie 2.1.0, were downloaded to the King's College London High Performance Computer Cluster Rosalind, using the synapse client (1.7.5). Bam files were merged, and fastq files were extracted using samtools $1.5^{62}$ and the flag '-F 0x100'. Trimmomatic $0.38^{63}$ was used to prune Illumina adaptors, low quality bases (leading/trailing sequences with phred score $<3$, or those with average score $<15$ every four bases), or reads below 36 bases in length. Trimmed reads were mapped to the human genome hg38 using bowtie ${ }^{64}$ and the parameters -very-sensitive-local, -k 100, and --score-min L,0,1.6. Subsequently, Telescope 1.0.2 was used to quantify expression of 14,968 HERVs (annotation version hg38), which we defined as the retrogenome ${ }^{20}$. We analyzed HERVs with counts $>10$ across 4 samples at least, to avoid inflation driven by lowly expressed elements. Case-control expression differences ( $N=538$ individuals in total) were calculated in $\mathrm{R}^{65}$ using Wald tests in DESeq26 ${ }^{66}$, where data was normalized (median of ratios) and controlled for the main confounders of gene expression estimated by Fromer and colleagues $^{39}$, which included institution of sample origin, RNA integrity number, gender, post-mortem interval, age (determined in five bins: \#1 = 13-29 years, \#2 = 30-49 years, \#3 = 50-69 years, \#4 = 70-89 years, $\# 5=90+$ years), as well as the first five population covariates estimated using multidimensional scaling in PLINK 1.967, and the first ten hidden HERV expression confounders estimated using sva ${ }^{68}$, considering schizophrenia and unaffected individuals only.

\section{Whole-genome genotype data processing}

Markers with zero alternate alleles, genotyping call rate $<0.98$, Hardy-Weinberg $P<5 \times 10^{-5}$, or individuals with genotyping call rate $<0.90$, were removed from the analysis, as described by Fromer and colleagues ${ }^{39}$. PLINK files were generated containing genotype information for 958,178 variants for the 593 subjects. Marker alleles were phased to the forward strand, and ambiguously stranded 
bioRxiv preprint doi: https://doi.org/10.1101/798017; this version posted October 8, 2019. The copyright holder for this preprint (which was not certified by peer review) is the author/funder, who has granted bioRxiv a license to display the preprint in perpetuity. It is made available under aCC-BY-NC 4.0 International license.

markers were removed. Additional genotype information was imputed from the 1000 Genomes Phase 1 reference panel using minimac3 and Eagle v2.3 phasing with the Michigan Imputation Server (https://imputationserver.sph.umich.edu/index.html). Genotype information from the 22 autosomes was concatenated using bcftools 1.9 (https://samtools.github.io/bcftools/bcftools.html), non-single nucleotide polymorphisms (SNPs) were excluded, as well as sites with an imputation $\mathrm{R}^{2}$ $<0.8$, minor allele frequency $<0.05$, or Hardy-Weinberg $\mathrm{P}<5 \times 10^{-5}$.

\section{eQTL analysis}

Normalized HERV counts per sample were obtained using DESeq2 $(N=593)$, and HERV expression was tested for the effect of genotype at all variants located within a $1 \mathrm{Mb}$ window upstream or downstream from the annotation start site of each HERV using QTLtools ${ }^{69}$, according to the authors' manual. We covaried for the effect of case-control status, institution of sample origin, RNA integrity number, gender, post-mortem interval, age (five bins, as described previously), the first five population covariates, and ten hidden expression confounders estimated using sva ${ }^{68}$ (Supplemental Figure 3). eQTL P-values were corrected through estimation of a beta distribution using a minimum of 1,000 permutations and maximum of 10,000 , and were further corrected for the number of HERVs tested using the false discovery rate method $(q<0.05)$.

\section{Weighted Correlation Network Analysis (WGCNA)}

WGCNA is a systems biology approach that enables the identification of co-expressed genes in transcriptomic data, which we used here to identify the genes co-expressed with schizophrenia HERVs in order to infer their biological function ${ }^{35}$. We used this tool to construct a signed network consisting of HERVs and genes, which was created based on an adjacency matrix that informs about the co-expression similarity observed between all pairs of genes and HERVs in the expression data (i.e. genes and genes, genes and HERVs, HERVs and HERVs). Normalized HERV and gene counts were variance-stabilized in DESeq2, and were further adjusted for all confounders previously described, using the removebatcheffect function in limma ${ }^{70}$. To achieve this, we combined gene and HERV counts obtained from the brain of 538 individuals (279 unaffected individuals and 259 schizophrenia cases), and filtered out genes and HERVs that were lowly expressed, i.e. those with 
bioRxiv preprint doi: https://doi.org/10.1101/798017; this version posted October 8, 2019. The copyright holder for this preprint (which was not certified by peer review) is the author/funder, who has granted bioRxiv a license to display the preprint in perpetuity. It is made available under aCC-BY-NC 4.0 International license.

$<10$ counts in $<80 \%$ of samples), as these can drive spurious correlations ${ }^{35}$. The normalized counts were variance stabilized transformed using DESeq2 and adjusted for institution of sample origin, gender, case-control status, age bins, post-mortem interval, the first five population dimensions (estimated in plink), and RIN, using limma ${ }^{70}$. WGCNA identifies modules by applying hierarchical clustering to the adjacency matrix, further filtering spurious relationships through the application of a topological overlap approach. We used an $R^{2}$ cut-off of 0.8 , which corresponds to a $\beta=12$, to construct the network. Each module was assigned a color, and genes or HERVs not belonging to any module were assigned to the gray module. The relationship between modules and specific cell types was tested based on the correlation between the module eigengenes (ME), defined as the first principal component of the module, and cell count estimates, as described below. We applied the false discovery rate method to correct for the module-cell type associations $(q<0.05)$. Plots were generated by WGCNA.

\section{Cell type estimates and module correlations}

We performed a BRain cEll Type speclfic Gene Expression Analysis (BRETIGEA) ${ }^{37}$ to estimate the abundance of major neural cell types in the 538 samples analyzed by WGCNA. Briefly, BRETIGEA uses expression data from single cell RNA-sequencing data sets to identify the proportion of astrocytes, microglia, endothelial cells, oligodendrocytes, oligodendrocyte progenitor cells and neurons, in bulk brain gene expression data. More specifically, this tool uses a panel of 50 wellestablished cell type-specific markers to generate coefficients that represent the proportion of each cell type per sample, which were tested for association with each module.

\section{Gene Ontology (GO) analyses}

We performed GO analyses using the WEB-based GEne SeT AnaLysis Toolkit (Webgestalt) ${ }^{71}$ to identify the function of the genes co-regulated with the schizophrenia HERVs in the brain, and thus infer the potential function of these HERVs. All genes inputted to WGCNA were used as background (reference) gene set. We used the false discovery rate method to correct for the GO enrichment analyses within Webgestalt $(q<0.05)$ and report up to 10 significant $\mathrm{GO}$ terms per module. Volcano plots were generated in Webgestalt. 


\section{Statistical analysis and data visualization}

516 The co-localization of GWAS-supported variants with the retrogenome was calculated using linear

517 regressions in GARFIELD ${ }^{27}$, and the gene-level and gene-set enrichment analyses were calculated

518 in MAGMA $^{28}$. Findings were corrected for multiple testing using the Bonferroni method. The case519 control HERV expression differences ( $\mathrm{N}=538$ individuals) and effects of eQTLs on HERV 520 expression ( $\mathrm{N}=593$ individuals) were calculated, respectively, using Wald tests in DESeq2, and 521 stepwise linear regressions in QTLtools, respectively. These were corrected using the false 522 discovery rate $(q<0.05)$, a more permissive multiple testing correction method, to increase our 523 detection power. The effect of genotype on specific HERVs within cases and control groups, 524 separately or combined, was calculated using linear regressions in IBM Statistics SPSS 25 (IBM 525 Corp., Armonk, NY, United States). Other analyses were performed in $\mathrm{R}^{65}$. Graphs were generated 526 in R or Graph Pad Prism 7 (GraphPad Software, San Diego, CA, United States). 
bioRxiv preprint doi: https://doi.org/10.1101/798017; this version posted October 8, 2019. The copyright holder for this preprint (which was not certified by peer review) is the author/funder, who has granted bioRxiv a license to display the preprint in perpetuity. It is made available under aCC-BY-NC 4.0 International license.

\section{Acknowledgements}

The work was supported in part by US National Institutes of Health grants: CA206488 (DFN), Al076059 (DFN) and UL1TR001876 (KAC). This work was also supported by a grant from the Coordenação de Aperfeiçoamento de Pessoal de Nível Superior (CAPES, Brazil, Science without Borders award no. BEX1279/13-0) and an NIHR Maudsley Biomedical Research Centre Career Development Award to RRRD. TRP is funded by a Medical Research Council (MRC) Skills Development Fellowship (MR/N014863/1). This study represents independent research part funded by the NIHR-Wellcome Trust King's Clinical Research Facility and the National Institute for Health Research (NIHR) Biomedical Research Centre at South London and Maudsley NHS Foundation Trust and King's College London. The views expressed are those of the authors and not necessarily those of the NHS, the NIHR or the Department of Health and Social Care. Data for this publication were obtained from NIMH Repository \& Genomics Resource, a centralized national biorepository for genetic studies of psychiatric disorders. Data were generated as part of the CommonMind Consortium supported by funding from Takeda Pharmaceuticals Company Limited, F. Hoffman-La Roche Ltd and NIH grants R01MH085542, R01MH093725, P50MH066392, P50MH080405, R01MH097276, RO1-MH-075916, P50M096891, P50MH084053S1, R37MH057881, AG02219, AG05138, MH06692, R01MH110921, R01MH109677, R01MH109897, U01MH103392, and contract HHSN271201300031C through IRP NIMH. Brain tissue for the study was obtained from the Mount Sinai NIH Brain and Tissue Repository, the University of Pennsylvania Alzheimer's Disease Core Center, the University of Pittsburgh NeuroBioBank and Brain and Tissue Repositories, and the NIMH Human Brain Collection Core. CMC Leadership: Panos Roussos, Joseph Buxbaum, Andrew Chess, Schahram Akbarian, Vahram Haroutunian (Icahn School of Medicine at Mount Sinai), Bernie Devlin, David Lewis (University of Pittsburgh), Raquel Gur, Chang-Gyu Hahn (University of Pennsylvania), Enrico Domenici (University of Trento), Mette A. Peters, Solveig Sieberts (Sage Bionetworks), Thomas Lehner, Stefano Marenco, Barbara K. Lipska (NIMH). Tissue samples used in RT-qPCR experiments were supplied by The London Neurodegenerative Diseases Brain Bank, which receives funding from the Medical Research Council and as part of the Brains for Dementia Research programme, jointly funded by Alzheimer's Research UK and Alzheimer's Society. We thank Professor Cathryn Lewis for her comments on the manuscript and Daniel Bean (King's College 
557 London) for his assistance with coding. We also thank Richard "Brad" Jones, Mario Ostrowski, and

558 Nathaniel Bachtel for their comments on this manuscript.

559

\section{Author contributions}

561 Study design: TRP, RRRD. Performed analyses and experiments: RRRD, TRP. Contributed 562 reagents, biological material, revised the manuscript: MLB, MM, CEO, GAB, SS, CT, GRT, KAC, 563 DPS, DFN. Wrote the paper: RRRD, TRP.

565 Conflict of Interest

566 The authors declare no conflict of interest.

568 Data availability

569 Telescope and the HERV annotation are available at http://github.com/mlbendall/telescope. Access 570 to the CommonMind Consortium dataset can be requested to the NIMH Repository \& Genomics 571 Resource via https://www.nimhgenetics.org/resources/commonmind. 
bioRxiv preprint doi: https://doi.org/10.1101/798017; this version posted October 8, 2019. The copyright holder for this preprint (which was not certified by peer review) is the author/funder, who has granted bioRxiv a license to display the preprint in perpetuity. It is made available under aCC-BY-NC 4.0 International license.

\section{References}

1. Gifford, R. \& Tristem, M. The evolution, distribution and diversity of endogenous retroviruses. Virus Genes 26, 291-315 (2003).

2. Lander, E.S. et al. Initial sequencing and analysis of the human genome. Nature 409, 860921 (2001).

3. Mi, S. et al. Syncytin is a captive retroviral envelope protein involved in human placental morphogenesis. Nature 403, 785-9 (2000).

4. Grow, E.J. et al. Intrinsic retroviral reactivation in human preimplantation embryos and pluripotent cells. Nature 522, 221-225 (2015).

5. Brattas, P.L. et al. TRIM28 Controls a Gene Regulatory Network Based on Endogenous Retroviruses in Human Neural Progenitor Cells. Cell Rep 18, 1-11 (2017).

6. Fasching, L. et al. TRIM28 Represses Transcription of Endogenous Retroviruses in Neural Progenitor Cells. Cell reports 10, 20-28 (2015).

7. Li, W. et al. Human endogenous retrovirus-K contributes to motor neuron disease. Sci Transl Med 7, 307ra153 (2015).

8. Douville, R., Liu, J., Rothstein, J. \& Nath, A. Identification of active loci of a human endogenous retrovirus in neurons of patients with amyotrophic lateral sclerosis. Annals of neurology 69, 141-151 (2011).

9. Garson, J.A. et al. Quantitative analysis of human endogenous retrovirus-K transcripts in postmortem premotor cortex fails to confirm elevated expression of HERV-K RNA in amyotrophic lateral sclerosis. Acta Neuropathol Commun 7, 45 (2019).

10. Perron, H. et al. Molecular characteristics of Human Endogenous Retrovirus type-W in schizophrenia and bipolar disorder. Transl Psychiatry 2, e201 (2012).

11. Weis, S. et al. Reduced expression of human endogenous retrovirus (HERV)-W GAG protein in the cingulate gyrus and hippocampus in schizophrenia, bipolar disorder, and depression. J Neural Transm (Vienna) 114, 645-55 (2007).

12. Yolken, R.H., Karlsson, H., Yee, F., Johnston-Wilson, N.L. \& Torrey, E.F. Endogenous retroviruses and schizophrenia. Brain Res Brain Res Rev 31, 193-9 (2000).

13. Roussos, P. et al. A role for noncoding variation in schizophrenia. Cell reports 9, 1417-1429 (2014).

14. Pardiñas, A.F. et al. Common schizophrenia alleles are enriched in mutation-intolerant genes and in regions under strong background selection. Nature Genetics 50, 381-389 (2018).

15. Schizophrenia Working Group of the Psychiatric Genomics Consortium et al. Biological insights from 108 schizophrenia-associated genetic loci. Nature 511, 421 (2014).

16. Goke, J. \& Ng, H.H. CTRL+INSERT: retrotransposons and their contribution to regulation and innovation of the transcriptome. EMBO Rep 17, 1131-44 (2016).

17. Karlsson, H. et al. Retroviral RNA identified in the cerebrospinal fluids and brains of individuals with schizophrenia. Proc Natl Acad Sci U S A 98, 4634-9 (2001).

18. Diem, O., Schäffner, M., Seifarth, W. \& Leib-Mösch, C. Influence of Antipsychotic Drugs on Human Endogenous Retrovirus (HERV) Transcription in Brain Cells. PLoS ONE 7, e30054 (2012).

19. Svensson, A.C. et al. Chromosomal distribution, localization and expression of the human endogenous retrovirus ERV9. Cytogenet Cell Genet 92, 89-96 (2001).

20. Bendall, M.L. et al. Telescope: Characterization of the retrotranscriptome by accurate estimation of transposable element expression. PLOS Computational Biology In press, 398172 (2018).

21. Subramanian, R.P., Wildschutte, J.H., Russo, C. \& Coffin, J.M. Identification, characterization, and comparative genomic distribution of the HERV-K (HML-2) group of human endogenous retroviruses. Retrovirology 8, 90-90 (2011). 
bioRxiv preprint doi: https://doi.org/10.1101/798017; this version posted October 8, 2019. The copyright holder for this preprint (which was not certified by peer review) is the author/funder, who has granted bioRxiv a license to display the preprint in perpetuity. It is made available under aCC-BY-NC 4.0 International license.

623 22. Grandi, N., Cadeddu, M., Blomberg, J. \& Tramontano, E. Contribution of type W human endogenous retroviruses to the human genome: characterization of HERV-W proviral insertions and processed pseudogenes. Retrovirology 13, 67-67 (2016).

23. Grandi, N. et al. Identification of a novel HERV-K(HML10): comprehensive characterization and comparative analysis in non-human primates provide insights about HML10 proviruses structure and diffusion. Mobile DNA 8, 15 (2017).

24. Vargiu, L. et al. Classification and characterization of human endogenous retroviruses; mosaic forms are common. Retrovirology 13, 7 (2016).

25. Tokuyama, M. et al. ERVmap analysis reveals genome-wide transcription of human endogenous retroviruses. Proceedings of the National Academy of Sciences 115, 1256512572 (2018).

26. Jeong, H.-H., Yalamanchili, H.K., Guo, C., Shulman, J.M. \& Liu, Z. An ultra-fast and scalable quantification pipeline for transposable elements from next generation sequencing data. in Biocomputing 2018 168-179.

27. Iotchkova, V. et al. GARFIELD classifies disease-relevant genomic features through integration of functional annotations with association signals. Nature Genetics 51, 343-353 (2019).

28. de Leeuw, C.A., Mooij, J.M., Heskes, T. \& Posthuma, D. MAGMA: Generalized Gene-Set Analysis of GWAS Data. PLOS Computational Biology 11, e1004219 (2015).

29. Bao, W., Kojima, K.K. \& Kohany, O. Repbase Update, a database of repetitive elements in eukaryotic genomes. Mobile DNA 6, 11 (2015).

30. Wang, D. et al. Comprehensive functional genomic resource and integrative model for the human brain. Science 362(2018).

31. Anderson, G.W. et al. Characterisation of neurons derived from a cortical human neural stem cell line CTX0E16. Stem Cell Res Ther 6, 149 (2015).

32. Deans, P.J.M. et al. Psychosis Risk Candidate ZNF804A Localizes to Synapses and Regulates Neurite Formation and Dendritic Spine Structure. Biol Psychiatry 82, 49-61 (2017).

33. Duarte, R.R.R. et al. The Psychiatric Risk Gene NT5C2 Regulates Adenosine Monophosphate-Activated Protein Kinase Signaling and Protein Translation in Human Neural Progenitor Cells. Biol Psychiatry 86, 120-130 (2019).

34. Hinrichs, A.S. et al. The UCSC Genome Browser Database: update 2006. Nucleic Acids Res 34, D590-8 (2006).

35. Langfelder, P. \& Horvath, S. WGCNA: an R package for weighted correlation network analysis. BMC Bioinformatics 9, 559 (2008).

36. van Dam, S., Vosa, U., van der Graaf, A., Franke, L. \& de Magalhaes, J.P. Gene coexpression analysis for functional classification and gene-disease predictions. Brief Bioinform 19, 575-592 (2018).

37. McKenzie, A.T. et al. Brain Cell Type Specific Gene Expression and Co-expression Network Architectures. Scientific Reports 8, 8868 (2018).

38. Klag, T. et al. Human Endogenous Retroviruses: Residues of Ancient Times Are Differentially Expressed in Crohn's Disease. Inflammatory Intestinal Diseases 3, 125-137 (2018).

39. Fromer, M. et al. Gene expression elucidates functional impact of polygenic risk for schizophrenia. Nature neuroscience 19, 1442-1453 (2016).

40. O'Brien, H.E. et al. Expression quantitative trait loci in the developing human brain and their enrichment in neuropsychiatric disorders. Genome biology 19, 194-194 (2018).

41. Bray, N.J. \& O'Donovan, M.C. The genetics of neuropsychiatric disorders. Brain and Neuroscience Advances 2, 2398212818799271 (2018).

42. Duarte, R.R.R. et al. Genome-wide significant schizophrenia risk variation on chromosome 10q24 is associated with altered cis-regulation of BORCS7, AS3MT, and NT5C2 in the human brain. Am J Med Genet B Neuropsychiatr Genet 171, 806-14 (2016). 
bioRxiv preprint doi: https://doi.org/10.1101/798017; this version posted October 8, 2019. The copyright holder for this preprint (which was not certified by peer review) is the author/funder, who has granted bioRxiv a license to display the preprint in perpetuity. It is made available under aCC-BY-NC 4.0 International license.

675 43. Liu, W. et al. Construction and Analysis of Gene Co-Expression Networks in Escherichia

44. van Dam, S., Võsa, U., van der Graaf, A., Franke, L. \& de Magalhães, J.P. Gene coexpression analysis for functional classification and gene-disease predictions. Briefings in Bioinformatics 19, 575-592 (2017).

45. Inkeles, M.S. et al. Cell-type deconvolution with immune pathways identifies gene networks of host defense and immunopathology in leprosy. JCI Insight 1(2016).

46. Tansey, K.E. \& Hill, M.J. Enrichment of schizophrenia heritability in both neuronal and glia cell regulatory elements. Translational Psychiatry 8, 7-7 (2018).

47. Hill, M.J. et al. Knockdown of the schizophrenia susceptibility gene TCF4 alters gene expression and proliferation of progenitor cells from the developing human neocortex. Journal of psychiatry \& neuroscience : JPN 42, 181-188 (2017).

48. Hill, M.J., Jeffries, A.R., Dobson, R.J., Price, J. \& Bray, N.J. Knockdown of the psychosis susceptibility gene ZNF804A alters expression of genes involved in cell adhesion. Hum Mol Genet 21, 1018-24 (2012).

49. Yengo, L. et al. Meta-analysis of genome-wide association studies for height and body mass index in approximately 700000 individuals of European ancestry. Hum Mol Genet 27, 36413649 (2018).

50. Harst, P.v.d. \& Verweij, N. Identification of 64 Novel Genetic Loci Provides an Expanded View on the Genetic Architecture of Coronary Artery Disease. Circulation Research 122, 433-443 (2018).

51. de Lange, K.M. et al. Genome-wide association study implicates immune activation of multiple integrin genes in inflammatory bowel disease. Nat Genet 49, 256-261 (2017).

52. Xue, A. et al. Genome-wide association analyses identify 143 risk variants and putative regulatory mechanisms for type 2 diabetes. Nature Communications 9, 2941 (2018).

53. Baselmans, B.M.L. et al. Multivariate genome-wide analyses of the well-being spectrum. Nature Genetics 51, 445-451 (2019).

54. The Early Genetics Lifecourse Epidemiology Eczema Consortium et al. Multi-ancestry genome-wide association study of 21,000 cases and 95,000 controls identifies new risk loci for atopic dermatitis. Nature Genetics 47, 1449 (2015).

55. Wray, N.R. et al. Genome-wide association analyses identify 44 risk variants and refine the genetic architecture of major depression. Nature Genetics 50, 668-681 (2018).

56. Stahl, E.A. et al. Genome-wide association study identifies 30 Loci Associated with Bipolar Disorder. bioRxiv, 173062 (2018).

57. Lambert, J.C. et al. Meta-analysis of 74,046 individuals identifies 11 new susceptibility loci for Alzheimer's disease. Nat Genet 45, 1452-8 (2013).

58. Demontis, D. et al. Discovery of the first genome-wide significant risk loci for attention deficit/hyperactivity disorder. Nature Genetics 51, 63-75 (2019).

59. van Rheenen, W. et al. Genome-wide association analyses identify new risk variants and the genetic architecture of amyotrophic lateral sclerosis. Nat Genet 48, 1043-8 (2016).

60. Grove, J. et al. Identification of common genetic risk variants for autism spectrum disorder. Nature Genetics 51, 431-444 (2019).

61. Turner, S.D. qqman: an R package for visualizing GWAS results using Q-Q and manhattan plots. bioRxiv, 005165 (2014).

62. Li, H. et al. The Sequence Alignment/Map format and SAMtools. Bioinformatics 25, 20782079 (2009).

63. Bolger, A.M., Lohse, M. \& Usadel, B. Trimmomatic: a flexible trimmer for Illumina sequence data. Bioinformatics 30, 2114-2120 (2014).

64. Langmead, B. \& Salzberg, S.L. Fast gapped-read alignment with Bowtie 2. Nature Methods 9, 357 (2012).

65. R Core Team. R: A language and environment for statistical computing. R Foundation for Statistical Computing, Vienna, Austria. (2018). 
bioRxiv preprint doi: https://doi.org/10.1101/798017; this version posted October 8, 2019. The copyright holder for this preprint (which was not certified by peer review) is the author/funder, who has granted bioRxiv a license to display the preprint in perpetuity. It is made available under aCC-BY-NC 4.0 International license.

66. Love, M.I., Huber, W. \& Anders, S. Moderated estimation of fold change and dispersion for RNA-seq data with DESeq2. Genome Biol 15, 550 (2014).

67. Chang, C.C. et al. Second-generation PLINK: rising to the challenge of larger and richer datasets. Gigascience 4, 7 (2015).

68. Jeffrey T. Leek et al. sva: Surrogate Variable Analysis. $R$ package Version 3.30.1, https://bioconductor.org/packages/release/bioc/html/sva.html(2019).

69. Delaneau, O. et al. A complete tool set for molecular QTL discovery and analysis. Nature Communications 8, 15452 (2017).

70. Ritchie, M.E. et al. limma powers differential expression analyses for RNA-sequencing and microarray studies. Nucleic Acids Research 43, e47-e47 (2015).

71. Wang, J., Vasaikar, S., Shi, Z., Greer, M. \& Zhang, B. WebGestalt 2017: a more comprehensive, powerful, flexible and interactive gene set enrichment analysis toolkit. Nucleic acids research 45, W130-W137 (2017). 
Contribution of polymorphisms in HERVs to selected traits

Significance

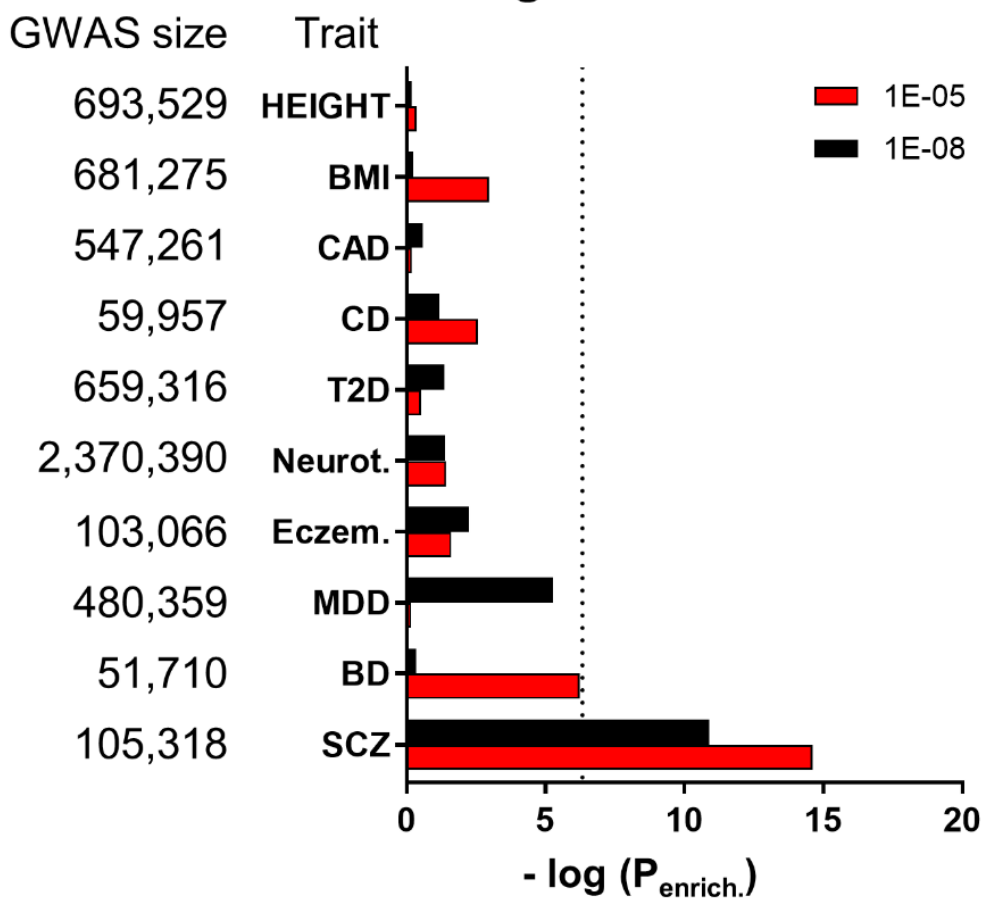

Effect

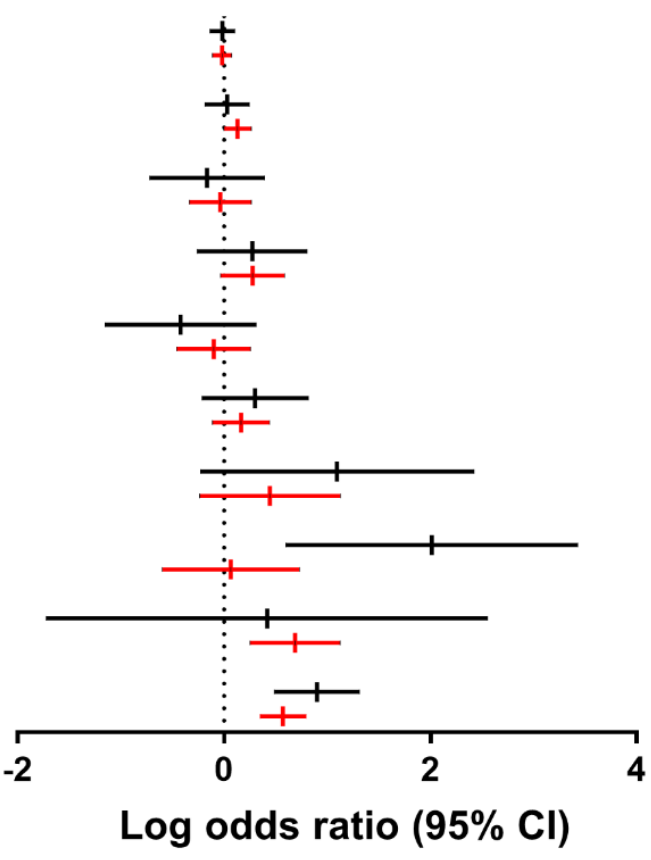

Figure 1. Variants within the retrogenome are enriched with schizophrenia-associated 744 polymorphisms. No association with any other trait was observed (see Supplemental Table 1 for 745 full results). Calculated using GARFIELD ${ }^{27}$. 
(A) Gene-level Q-Q plot

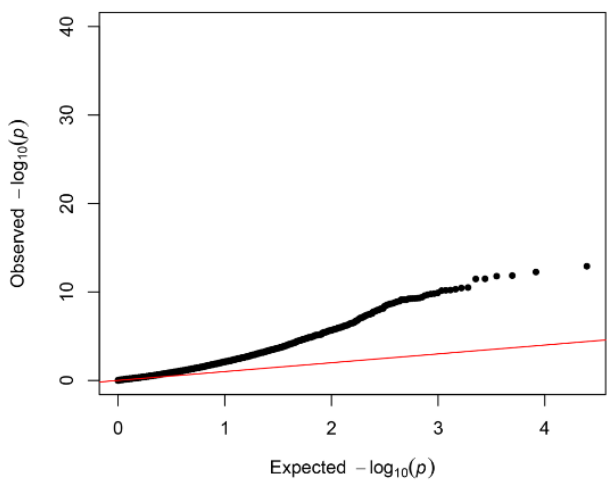

(B) Gene-level Manhattan plot

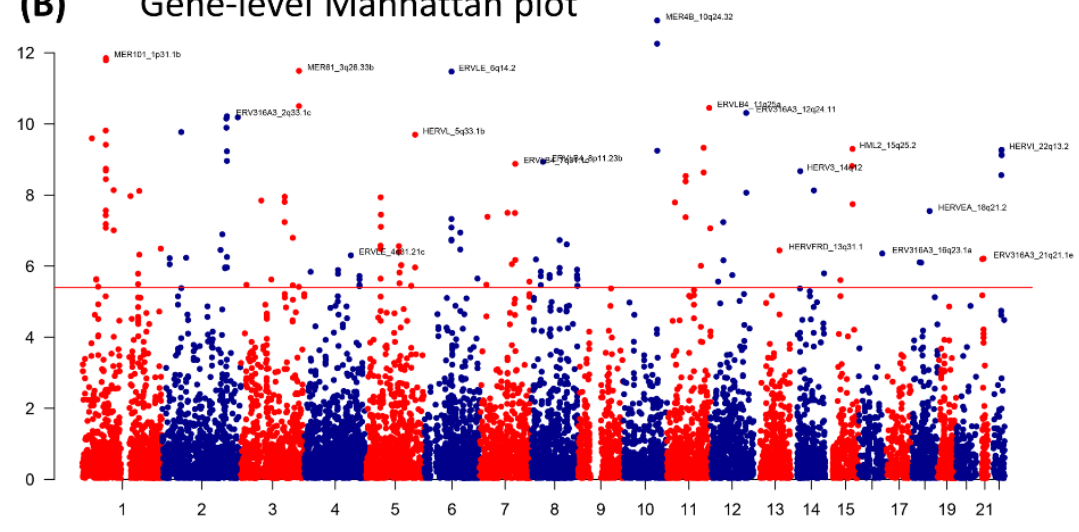

749 Figure 2. Gene-level enrichment analysis of the schizophrenia GWAS summary statistics using the 750 HERV annotation developed by Bendall and colleagues ${ }^{20}$, calculated using MAGMA $^{28}$. 751 Chromosomes 1-22 only, extended MHC region excluded (chromosome 6, from 26-34 Mb). (A) 752 Quantile-quantile plot showing the contribution of several HERVs to schizophrenia genetics 753 compared to an expected normal distribution (red line). (B) Manhattan plot showing the location of 754 the HERVs associated with schizophrenia. Plots created using qqman ${ }^{61}$. All enriched HERVs are shown on Supplemental Table 2. 
(A)

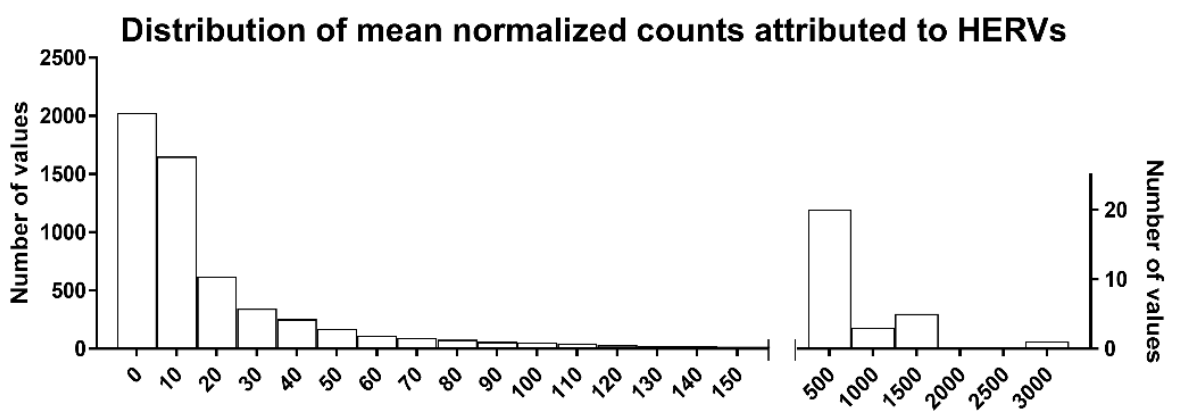

(B)

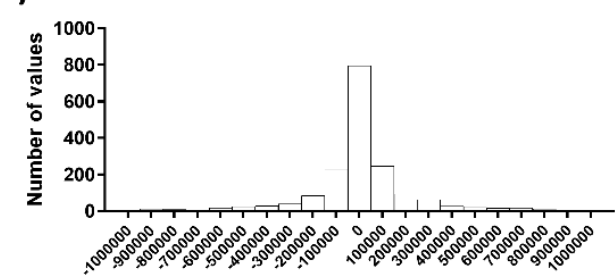

(C)

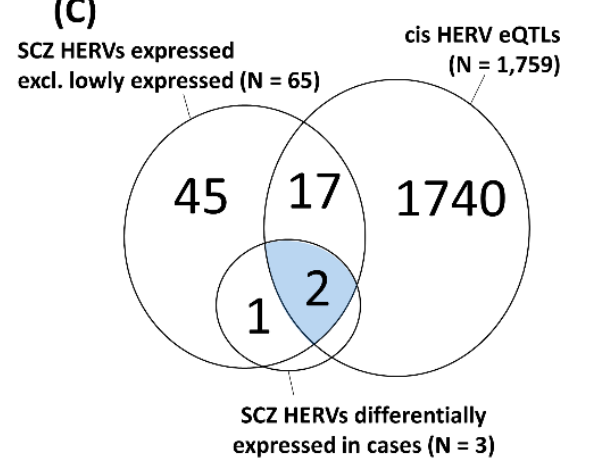

(D)

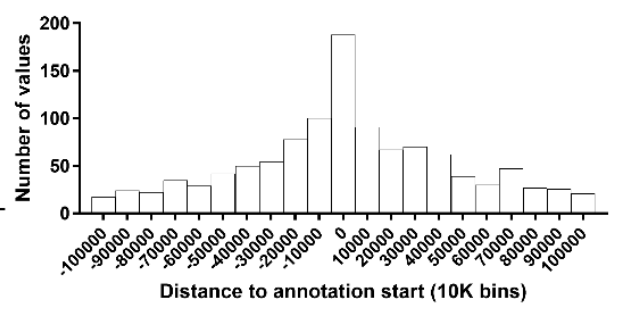

Figure 3. HERV expression in the dorsolateral prefrontal cortex, based on an analysis of the

CommonMind Consortium dataset with Telescope. (A) Frequency distribution of the mean normalized counts per HERV across samples showing that the majority of HERVs are lowly expressed, according to an analysis of 593 post-mortem brains. Normalized counts do not include adjustments utilized in the analyses. (B) Distribution of values representing the distance between the eQTL for a HERV and the start site for that HERV. Left panel shows all data in bins of 100,000, and the right panel only data nearer the start site of the HERVs, in bins of 10,000 . A large proportion of eQTLs was located within a 10kb window upstream or downstream the start coordinates of the HERV they regulate. (C) Overlap between the 65 schizophrenia-associated HERVs expressed in the brain, the 1,759 HERVs modulated by eQTLs, and the three HERVs enriched for schizophrenia variants and additionally differentially expressed between schizophrenia cases $(N=259)$ and unaffected individuals ( $N=279$ ). (D) Heatmap of the six HERVs enriched for schizophrenia variants and further differentially expressed in patients, separated by gender. In blue, HERVs that are modulated by eQTLs, as demonstrated in Figure 4. 
(A) Controls ( $\mathrm{N}=279) \times \mathrm{SCZ}(\mathrm{N}=259)$

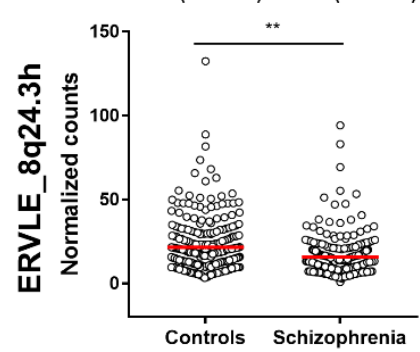

(B) Controls (N=279) $\times$ SCZ (N=259)

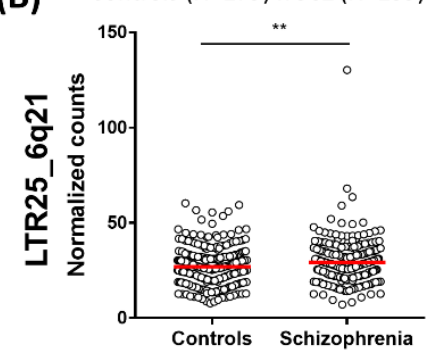

All individuals $(\mathrm{N}=593)$

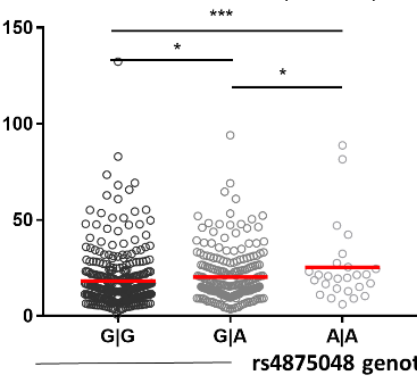

All individuals $(\mathrm{N}=593)$

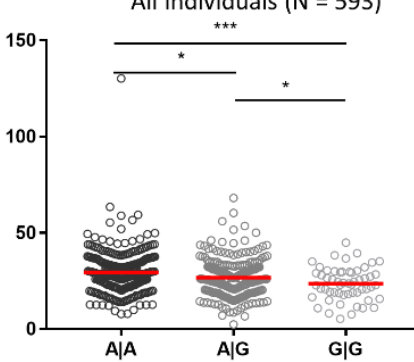

Controls only $(\mathrm{N}=279)$

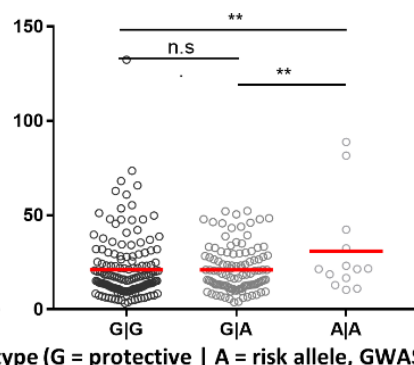

Controls only $(\mathrm{N}=279)$

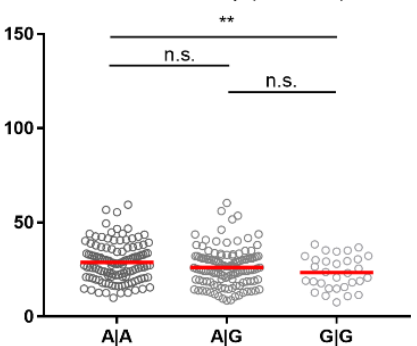

SCZ cases only $(\mathrm{N}=259)$

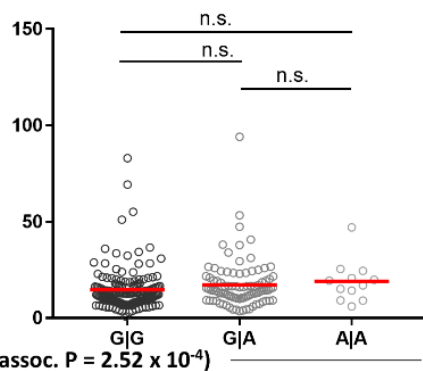

SCZ cases only $(\mathrm{N}=259)$

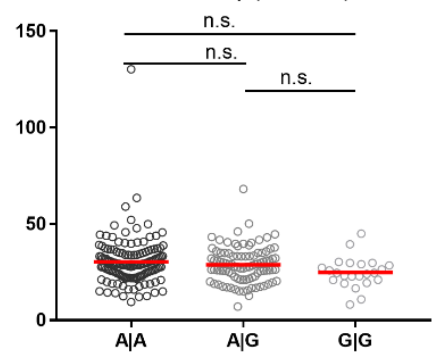

_rs174399 genotype (in LD with $r s 11153302\left[R^{2}=0.4, D^{\prime}=1.0\right.$, GWAS $\left.P=6.4 \times 10^{-5}\right]$ : $A=$ protective $\mid G=$ risk allele)

774 Figure 4. HERVs enriched for schizophrenia variants and differentially expressed between cases

775 and controls, and according to genotype. eQTLs modulated HERV expression exclusively in control

776 individuals. Graphs show normalized counts associated with case-control status (left; Wald tests, $\left.{ }^{* *} \mathrm{P}<0.01\right)$ or per genotype considering all individuals, control individuals or schizophrenia patients only (last three graphs, respectively; ANOVAs followed by pairwise comparisons corrected using the

779 Bonferroni method $\left({ }^{*} P<0.05,{ }^{* *} P<0.01,{ }^{* *} P<0.001\right.$; n.s.: not significant; Supplemental Table

780 6). Data shown are for (A) ERVLE_8q24.3h and its top eQTL in the DLPFC, rs4875048, and (B)

781 LTR25_6q21 and its top eQTL, rs174399. Values are uncorrected for the factors and covariates

782 included in the eQTL analysis. 
(A) Gene Ontology analysis
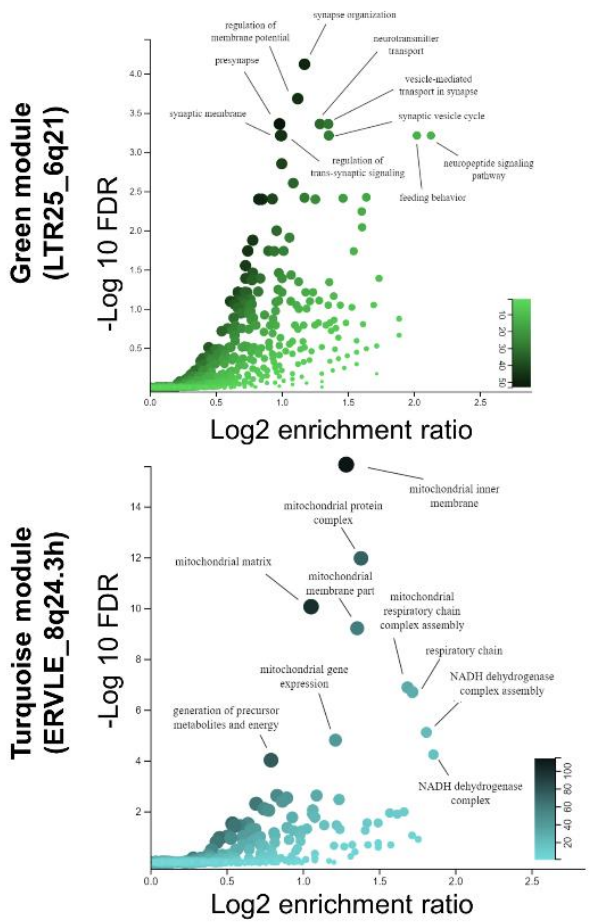

(B) Module-trait relationships

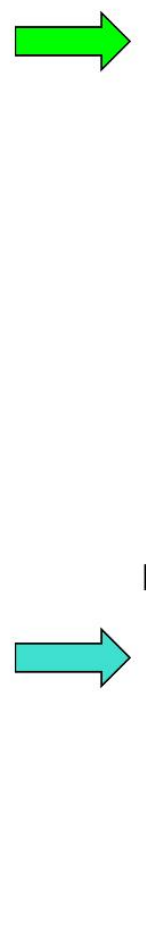

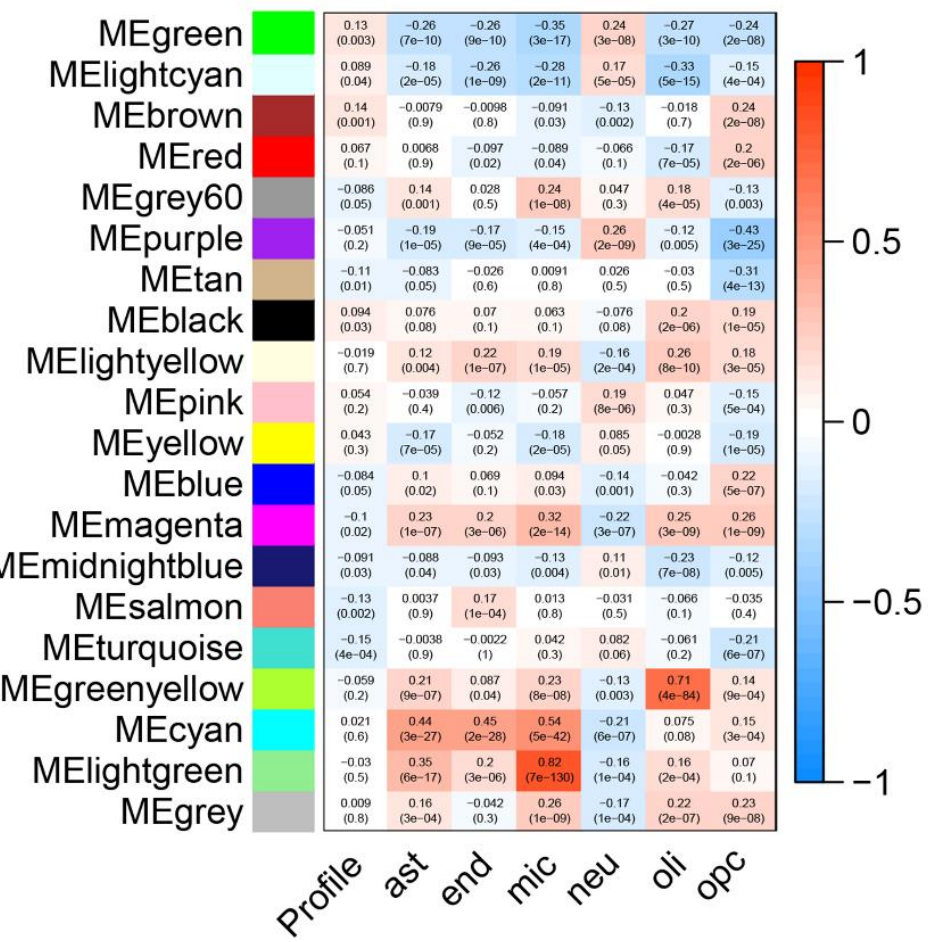

Figure 5. Gene ontology enrichment analysis of the co-expression modules associated with the green (LTR25_6q21) and turquoise modules (ERVLE_8q24.3h), and correlations between modules eigengenes, case-control status (Profile) and coefficients associated with cell counts for major neural cell types. (A) LTR25_6q21 belongs to the green module, which is significantly enriched for GO terms associated with neuronal function, as shown in the Volcano plot. ERVLE_8q24.3h, in turn, belongs to the turquoise module, which is significantly enriched for GO terms associated with mitochondrial function. (B) The green module is positively associated with neuronal counts, whereas the turquoise module does not correlate strongly with a specific cell type, apart from a negative correlation with oligodendrocyte progenitor cell counts. Each cell of the heatmap contains the Pearson's r coefficient followed by significance of the correlation $(P)$. Ast: astrocytes, end: endothelial cells, mic: microglia, neu: neurons, oli: oligodendrocytes, opc: oligodendrocyte progenitor cells. 


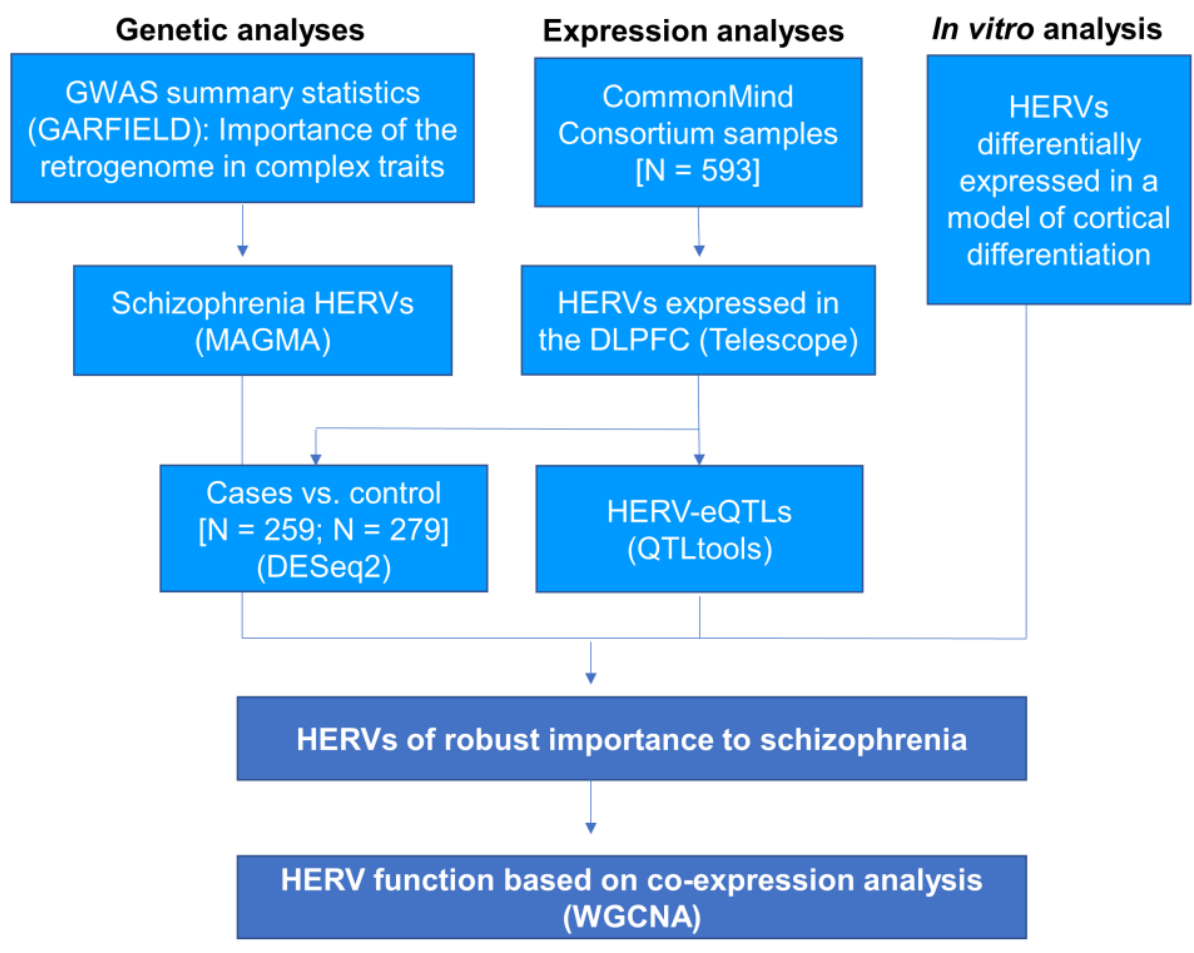

798 Figure 6. Analysis strategy. We performed a series of analyses using post-mortem brain RNA-

799 sequencing data, genetic enrichment analyses and in vitro cortical differentiation data to identify

800 HERVs of robust importance in schizophrenia. Bioinformatic tools used are indicated in

801 parentheses. DLPFC: dorsolateral prefrontal cortex. 
Table 1. Association of the eQTLs (and variants in linkage disequilibrium) with schizophrenia.

\begin{tabular}{|c|c|c|c|c|c|c|c|c|c|c|c|c|}
\hline HERV & SNP & Chromosome & Position & $\begin{array}{l}\text { Risk } \\
\text { allele }\end{array}$ & $\begin{array}{l}\text { Other } \\
\text { allele }\end{array}$ & $\begin{array}{l}\text { Frq } \\
\text { cases }\end{array}$ & OR & SE & $\mathbf{P}$ & $\mathbf{R}^{2 \neq}$ & $D^{\prime *}$ & \\
\hline \multirow[t]{2}{*}{ ERVLE_8q24.3h } & rs4875048 & 8 & 144826671 & $A$ & $G$ & 0.1869 & 1.0553 & 0.011855 & 5.6E-06 & - & - & \\
\hline & rs 10552126 & 8 & 144844056 & CAT & $\mathrm{C}$ & 0.2018 & 1.05919 & 0.0116 & 7.1E-07 & 0.8954 & & 1 \\
\hline \multirow[t]{2}{*}{ LTR25_6q21 } & rs174399 & 6 & 111919619 & $\mathrm{G}^{*}$ & A & 0.2565 & 1.0025 & 0.010488 & 0.81 & - & - & \\
\hline & rs11153302 & 6 & 111918869 & $A$ & $\mathrm{G}$ & 0.506 & 1.0397 & 0.00973 & $6.4 \mathrm{E}-05$ & 0.38 & & 1 \\
\hline
\end{tabular}

* This SNP is not associated with schizophrenia, but is in linkage disequilibrium with another variant that is associated with this disorder.

$¥$ Linkage disequilibrium statistics in relation to the top association signal at the locus which is in LD with the HERV-eQTL. 